\title{
OCORRÊNCIA DE IXODIDAE PARASITOS DE CAPIVARA (Hydrochoerus hydrochaeris LINNAEUS, 1766) NA ESTAÇÃO ECOLÓGICA DO TAIM, RIO GRANDE - RS, BRASIL ${ }^{1}$
}

\author{
OCCURENCE OF IXODIDAE PARASITES OF CAPYBARA (Hydrochoeurus \\ hydrochaeris LINNAEUS, 1766) IN THE ECOLOGIC AREA \\ OF TAIM, RIO GRANDE - RS, BRAZIL
}

\begin{abstract}
Afonso Lodovico Sinkoc ${ }^{2}$ João Guilherme Werner Brum ${ }^{3}$ Gertrud Müller ${ }^{4}$ Angela Begrow ${ }^{5}$ Rosa Maria Massaro Paulsen ${ }^{6}$
\end{abstract}

RESUMO

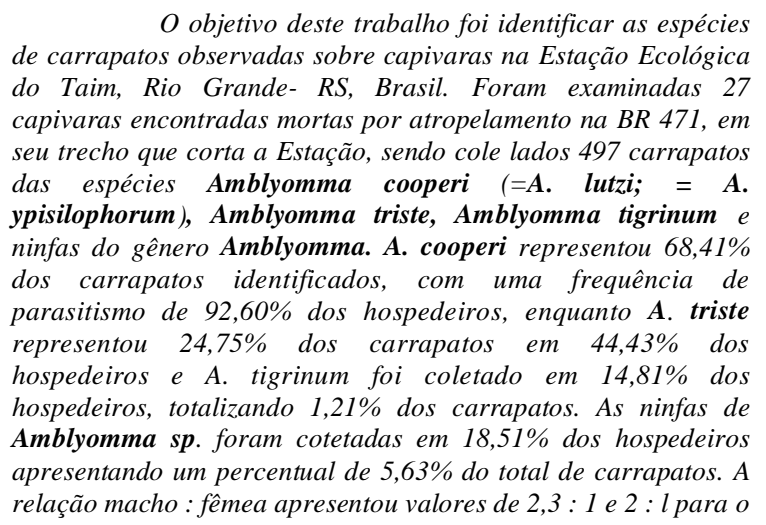

O objetivo deste trabalho foi identificar as espécies de carrapatos observadas sobre capivaras na Estação Ecológica do Taim, Rio Grande- RS, Brasil. Foram examinadas 27 capivaras encontradas mortas por atropelamento na BR 471, em seu trecho que corta a Estação, sendo cole lados 497 carrapatos das espécies Amblyomma cooperi $(=A$. lutzi; $=A$. ypisilophorum), Amblyomma triste, Amblyomma tigrinum $e$ ninfas do gênero Amblyomma. A. cooperi representou $68,41 \%$ dos carrapatos identificados, com uma frequência de parasitismo de $92,60 \%$ dos hospedeiros, enquanto A. triste representou $24,75 \%$ dos carrapatos em $44,43 \%$ dos hospedeiros e A. tigrinum foi coletado em $14,81 \%$ dos hospedeiros, totalizando $1,21 \%$ dos carrapatos. As ninfas de Amblyomma sp. foram cotetadas em 18,51\% dos hospedeiros apresentando um percentual de 5,63\% do total de carrapatos. A relação macho : fêmea apresentou valores de 2,3: 1 e 2 : l para o

\begin{abstract}
A. cooperi $\boldsymbol{e}$ A. triste, respectivamente. Todos hospedeiros apresentavam-se parasitados, com uma média de 18,4 carrapatos, variando de 1 a 64 carrapatos coletados por hospedeiro.

Palavras-chave: capivara, Hydrochoerus hydrochaeris, Amblyomma cooperi, Amblyomma triste. Amblyomma tigrinum, roedores, carrapatos, animais silvestres.
\end{abstract}

\section{SUMMARY}

The aim of this article was the identification ofthe tick species observed on capybara in wildlife in the Ecologic Area of Taim, Rio Grande - RS; Brazil. Twenly seven capybaras found dead by trample in the road BR 471 in the Ecologic Area, were

\footnotetext{
${ }^{1}$ Trabalho desenvolvido com o apoio do Instituto Brasileiro de Meio Ambiente e Recursos Naturais Renováveis (IBAMA), Fundação de Amparo à Pesquisa do Estado do Rio Grande do Sul (FAPERGS) e do Conselho Nacional de Desenvolvimento Científico e Tecnológico $(\mathrm{CNPq})$.

${ }^{2}$ Médico Veterinário, Pós-Graduando em Ciências Veterinárias (Área de Conselho - Doenças Parasitárias) Faculdade de Veterinária, Universidade Federal do Rio Grande do Sul.

${ }^{3}$ Médico Veterinário, MsC., PhD., Professor Titular, Departamento de Microbiologia e Parasitologia (DMP), Instituto de Biologia (IB), Universidade Federal de Pelotas (UFPel), Caixa Postal 354 - Campus Universitário, 96010-900 - Pelotas - RS. Autor para correspondência.

${ }^{4}$ Médico Veterinário, MSc., Professora Titular, DMP-IB-UFPel.

${ }^{5}$ Acadêmico de Medicina Veterinária, Faculdade de Veterinária, UFPel.

${ }^{6}$ Médico Veterinário, Bolsista de Aperfeiçoamento, CNPq.
} 
examined ticks coilected of the species Amblyomma cooperi (=A. tutu and = A. ypisilophorum), Amblyomma triste, Amblyomma tigrinum and ninphs of the Genus Amblyomma. Amblyomma cooperi represented $68.41 \%$ of the ticks with a frequency of parasitism of $92.60 \%$ of the hosts; Amblyomma triste species represented $24.75 \%$ of the coilected ticks $m 44.43 \%$ of the hosts; Amblyomma tigrinum species were coilected $m 14.81 \%$ of the hosts from a total of $1.21 \%$ of the found ticks and, the nimphs of Amblyomma were coilected in $18.51 \%$ of the hosts per forming $5.63 \%$ of the ali ticks coilected. The relation mate female showed values of 2.3:1 and 2:1 to A. cooperiandA. triste, respectivety. Ali examined hosts were found wiih ticks, with a mean of 18.4 ticks per host

Key words: capybara, Hydrochoerus hydrochaeris, Amblyomma cooperi, Amblyomma triste, Amblyomma tigrinum, rodents, ticks, wild animais.

\section{INTRODUÇÃO}

A capivara é o maior roedor conhecido, com distribuição limitada às Américas Central e do Sul (NOWAK \& PARADISO, 1986), sendo bastante comum no Rio Grande do Sul (SILVA, 1994). Possui um grande potencial para a exploração económica (ALHO, 1986; SILVA NETO, 1995) porém são escassas as informações sobre os parasites e as parasitoses que acometem este roedor, tanto em natureza quanto em cativeiro.

ARAGÃO (1936) citou o parasitismo da capivara pêlos carrapatos Amblyomma brasiliense, Amblyomma parvum e Amblyomma striatum; citou ainda que o Amblyomma cooperi é a espécie de carrapato típica da capivara na América do Sul, podendo parasitar, as vezes, a Anta (Tapirus terrestris); segundo este autor, esta espécie apresenta como área de ocorrência o sul da Bolívia, Paraguai, Argentina e, no Brasil, ocorre nos Estados do Paraná, São Paulo, Rio de Janeiro, Minas Gerais, Mato Grosso, Goiás e Pará.

$\mathrm{Na}$ Venezuela foram citadas originalmente a ocorrência do parasitismo em capivaras pêlos carrapatos A. cooperi (VOGELSANG \& CORDERO, 1940) e Anocentor nitens (VOGELSANG \& SANTOS DIAS, 1953). JONES et al., (1972) relacionaram para a capivara, na Venezuela, a ocorrência dos carrapatos Amblyomma cajennense, A. cooperi, A. coelebs, A. extraoculatum, A. oblongoguttatum, A. tigrinum e Amblyomma spp.; consideraram ainda que o $\boldsymbol{A}$. cooperi é um carrapato específico deste roedor e da Anta (Tapirus terrestris)

SCHULZE (1941) descreveu Amblyomma ypisilophorum coletado sobre capivaras no Estado de Santa Catarina. No Estado do Rio Grande do Sul,
CORREA (1954) referiu-se à ocorrência do A. triste parasitando cachorro doméstico no Município deJaguarão e A. cooperi parasitando a capivara, enquanto FREIRE (1967/68) relatou o achado de $\boldsymbol{A}$. triste sobre cachorro doméstico no município de Jaguarão. Posteriormente FREIRE (1972) identificou Amblyomma cajennense parasitando a capivara nos municípios de Herval do Sul e Jaguarão e, Amblyomma cooperi no município de Uruguaiana. Recentemente a ocorrência de Amblyomma cooperi em capivara foi observada no município de Santa Vitória do Palmar, RS (GONZALES \& OLIVEIRA, 1994).

KOHLS (1956) ata Amblyomma tigrinum sobre Pseudolapex sp. (Cachorro-doMato) no Rio Grande do Sul; sobre cachorro doméstico nos Estados do Rio Grande do Sul, Pará e Mato Grosso; sobre Tamanduá no Mato Grosso e sobre Chrysocyon sp. (Lobo-Guará) em Goiás. O autor citou ainda o Amblyomma triste coletado sobre vegetação no Noroeste do Mato Grosso, sobre Tapirus sp. (Anta) em localidade desconhecida e, no Estado do Pará sobre hospedeiro não identificado; afirmando serem estes os únicos registros, até então, conhecidos para esta espécie.

MACHADO et al. (1985) realizando estudos com o carrapato dos bovinos (Boophilus micropius) encontraram no Município de Aquidauana, no Estado do Mato Grosso do Sul o parasitismo simultâneo da capivara pêlos carrapatos Amblyomma parvum e Boophilus micropius.

$\mathrm{Na}$ Argentina GUGLIELMONE $\boldsymbol{e t}$ al. (1982) citaram o parasitismo de Amblyomma tigrinum em cão, gato, bovino, lebre, humano, e Cachorros-do- Mato (Cerdocyon sp. e Pseudolapex sp.), enquanto MAURI \& NAVONE (1993) registraram a ocorrência de $\boldsymbol{A}$. tigrinum sobre tatu (Chaetophractus villosus), na Província de Cordoba. O registro da ocorrência dos carrapatos Amblyomma cajennense, A. cooperi, A. dissimile, A. ovale e A. tigrinum em capivaras na Argentina foi feito por GUGLIELMONE \& VINABAL(1994).

O objetivo deste trabalho foi identificar as espécies de carrapatos que parasitam a capivara na Estação Ecológica do Taim (ESEC do Taim), bem como conhecer as espécies mais prevalentes.

\section{MATERIAIS E MÉTODOS}

As capivaras utilizadas neste estudo 462 foram recolhidas na BR 471, entre os quilómetros 462 e 508, no período de julho de 1994 a dezembro de 1995, no trecho que corta a ESEC do Taim, 
compreendendo os municípios de Rio Grande e Santa Vitória do Palmar, Estado do Rio Grande do Sul. Os animais foram transportados até a sede da ESEC do Taim, onde eram inspecionados na busca de ixodídeos, que foram coletados na totalidade. Os carrapatos foram removidos da pele do hospedeiro pela torção através do seu eixo longitudinal, fixados em água quente $\left(70^{\circ} \mathrm{C}\right)$ e conservados em frascos contendo álcool $70^{\circ} \mathrm{GL}$ glicerinado a $5 \%$. No laboratório os carrapatos foram analisados em lupa estereoscópica de mesa, sendo utilizadas para a identificação específica, as chaves propostas por ARAGÂO \& FONSECA (1961) e JONES et $\boldsymbol{a l}$. (1972). O resultado das identificações foram tabulados de acordo com a espécie, sexo e estado evolutivo do carrapato.

\section{RESULTADOS}

Foram coletados carrapatos de 27 animais, sendo identificadas 3 espécies: $\boldsymbol{A}$. cooperi Nuttall \& Warburton, 1908 (= Amblyomma lutzi Aragão, 1908 e A. ypsilophorum Schulze, 1941), A. tigrinum Koch, 1844 e A. triste Koch, 1844, apud ARAGÃO \& FONSECA (1961). De um total de 497 carrapatos coletados, o Amblyomma cooperi representou 68,41\%, o $\boldsymbol{A}$. triste $24,7{ }^{\prime} 5 \%$, о $\boldsymbol{A}$. tigrinum $1,21 \%$ e as ninfas somaram $5,63 \%$.

Infestações monoespecíficas estavam presentes em $37,03 \%$ dos hospedeiros, ocorrendo parasitismo por A. cooperi $(29,63 \%)$ e por A. triste (7,40\%); infestações por $\boldsymbol{A}$. cooperi associada a outra espécie estavam presentes em $55,55 \%$ dos hospedeiros, estando associado ao $\boldsymbol{A}$. triste (29,63\%), A. tigrinum (11,11\%) e a ninfas de Amblyomma (14,81\%). Infestações simultâneas de A. cooperi e A. triste associados a uma outra espécie foram observadas em 7,40\% dos animais, sendo a associação ao $\boldsymbol{A}$. tigrinum $(3,70 \%)$ e a ninfas de Amblyomma (3,70\%).

O parasitismo monoespecíflco por $\boldsymbol{A}$. cooperi totalizou $15,59 \%$ destes carrapatos coletados, variando de 1 a 16 espécimes por animal, com 6,63 carrapatos em média; quando associado a outras espécies, totalizou $84,41 \%$ dos espécimes, variando de 4 a 61 espécimes por animal com média de 16,88 carrapatos. O A. triste, quando coletado em parasitismos monoespecíficos, representou $26,83 \%$ do total coletado desta espécie, com o número total variando de 5 a 28 exemplares (média de 16,5); os exemplares desta espécie associados a outras espécies totalizaram $73,17 \%$, variando entre 1 e 42 exemplares, com média de 9 carrapatos por animal. O A. tigrinum ocorreu em 4 animais, sendo coletados 6 exemplares machos desta espécie.

A relação macho: fêmea apresentou valores de 2,3:1 e 2:1 para o A. cooperi e A. triste respectivamente. $\mathrm{O}$ numero de carrapatos por animal variou de 1 a 64 com uma média de 18,4, sendo que o A. cooperi variou de 1 a 63 com média de 13,6 carrapatos por animal, enquanto que o A. triste variou de 1 a 42 carrapatos com média de 10,25. O número de ninfas variou de 1 ali, com média de 5,6 ninfas por animal, sendo que nunca ocorreram isoladas e sim, sempre com pelo menos uma espécie de carrapato adulta.

\section{DISCUSSÃO}

A capivara pode ser parasitada por um elevado número de espécies de carrapatos (ARAGÃO, 1936; VOGELSANG \& SANTOS DIAS, 1953; FREIRE, 1972; JONES et al, 1972; MACHADO et al., 1985), sendo comum o parasitismo por espécies do género Amblyomma, e entre estas, o A. cooperi (ARAGÃO, 1936; VOGELSANG \& CORDERO, 1940; CORRÊA, 1954/55; FREIRE, 1972; JONES et al., 1972; GUGLIELMONE \& VINABAL, 1994; GONZALES \& OLIVEIRA, 1994), uma vez que este carrapato segundo ARAGÃO (1936), JONES et al (1972), GUGLIELMONE \& VINABAL (1994) parece ser específico da capivara, sem contudo, serem observadas informações adicionais sobre o status do parasitismo desta espécie de carrapato em Hydrochoerus hydrochaeris.

A ocorrência de $\boldsymbol{A}$. cooperi em Hydrochoerus hydrochaeris tem sido citada no RioGrande do Sul (CORRÊA, 1954/55; FREIRE, 1972; GONZALES \& OLIVEIRA, 1994), no Brasil (ARAGÃO, 1936; SCHULZE, 1941) e na América do Sul (ARAGÃO, 1936; VOGELSANG \& CORDERO,1940; JONES et al, 1972; GUGLIELMONE \& VINABAL, 1994). Na ESEC do Taim, o A. cooperi apresentou alta frequência de parasitismo nos hospedeiros $(92,59 \%)$ com uma média de 13,6 carrapatos por animal parasitado, totalizando $68,41 \%$ do total de carrapatos; confirmando assim as afirmações de especificidade desta espécie pela capivara (ARAGÃO, 1936; JONES et al., 1972; GUGLIELMONE \& VINABAL, 1994).

O A. triste apresentou níveis médios de frequência parasitaria $(44,44 \%)$ com 10,25 carrapatos em média por animal parasitado. A presença desta espécie no Rio Grande do Sul já foi citada por FREIRE (1967/68) sobre cachorro doméstico no Município de Jaguarão. IVANCOVICH (1980) citado 
por GUGLIELMONE \& VINABAL (1994) se refere à presença desta espécie na Argentina parasitando cães e o homem. Os índices de frequência parasitária e de coleta desta espécie encontrados na ESEC do Taim, indicam possivelmente uma perfeita adaptação à capivara como hospedeiro. Esta é a primeira citação desta espécie tendo a capivara como hospedeiro.

O A. tigrinum é um carrapato normalmente encontrado em carnívoros domésticos e, menos frequentemente em animais silvestres (KOHLS, 1956; GUGLIELMONE et al., 1982; GUGLIELMONE \& VINABAL, 1994). Foi descrito parasitando lebres, bovinos, homem e tatus na Argentina (GUGLIELMONE $\boldsymbol{e t}$ al., 1982; MAURI \& NAVONE, 1993), sendo que no Brasil, foi citado parasitando tamanduá (KHOLS, 1956) e o cervo-dopantanal Blastocerus dichotomus (SERRA-FREIRE et al, 1995) e, na Venezuela (JONES $\boldsymbol{e t}$ al, 1972) e Argentina (GUGLIELMONE \& VINABAL, 1994) em capivaras. Neste trabalho foi encontrado em 4 animais com um total de 6 exemplares machos. Como esta espécie representou apenas $1,26 \%$ do total de carrapatos coletados, considera-se a ocorrência desta espécie sobre a capivara como parasitismo acidental.

\section{AGRADECIMENTOS}

Os autores agradecem às Instituições financiadoras (FAPERGS, CNPq), ao Instituto Brasileiro do Meio Ambiente Recursos Naturais Renováveis (IBAMA), na pessoa dos Administradores da Estação Ecológica do Taim no período de coleta pela operacional ização da mesma e, ao Professor Carlos Marcos Barcelos de Oliveira pela revisão dos manuscritos.

\section{REFERÊNCIAS BIBLIOGRÁFICAS}

ALHO, C.J.R. Criação e manejo de capivaras em pequenas propriedades rurais. Brasília: EMBRAPA-DDT, 1986,48 p. (Série Documentos, 13).

ARAGÂO, H. de B. Ixodidas brasileiros e de alguns paízes limitrophes. Memórias do Instituto Oswaido Cruz, Rio de Janeiro, v. 31, n. 4, p. 759-844, 1936

ARAGÂO, H. de B., FONSECA, F. da. Notas de Ixodologia VIII. Lista e chave para os representantes da fauna ixodológica Brasileira. Memórias do Instituto Oswaido Cruz, Rio de Janeiro, v. 59, n. 2, p. 115-130, 1961

CORRÊA, O. Carrapatos determinados no Rio Grande do Sul Biologia, patologia e controle. Arquivos do Instituto de Pesquisas Veterinárias Desidério Finamor, Porto Alegre, v. 1., p. $35-50,1954 / 55$.

FREIRE, J.J. Fauna parasitária Riograndense. Revista da Faculdade de Agronomia e Veterinária, Porto Alegre, v. 9, p.111-149, 1967/68.

FREIRE, J.J. Revisão das espécies da família Ixodidae. Revista de Medicina Veterinária, São Paulo, v. 8, n. 1, p. 1-16, 1972
GONZALES, J.C., OLIVEIRA, C.M.B., Amblyomma cooperi em capivara, RS - Brasil. In: CONGRESSO DE MEDICINA VETERINÁRIA DO CONE SUL, 1., CONGRESSO ESTADUAL DE MEDICINA VETERINÁRIA, 12, 1994 Porto Alegre, RS. Anais... Porto Alegre : SOVERGS, 1994, p. 47.

GUGLIELMONE, A.A., MANGOLD, A.J., HADAN1, A. Amblyomma tigrinum Koch, 1844 en Ia Argentina. Su diagnóstico erróneo como Amblyomma maculatum y su distribuición geográfica. Gaceta Veterinária de Buenos Aires, v.44,n.367,p.57-63, 1982

GUGLIELMONE, A.A., VINABAL, A.E. Claves morfológicas dicotomicas e infonnacion ecológica para Ia identificacion de Ias garrapatas dei género Amblyomma Koch, 1844 de Ia Argentina. Revista Investigaciones Agropecuarias, Argentina, v. 25, n. 1, p. 39-47, 1994.

JONES, E.K., CLIFFORD, C.M., KEIRANS, J.E., et al., The ticks of Venezuela (Acarina : Ixodidoidea) with a key to the species of Amblyomma in the Westem Hemisphere. Brigham Young University Science Bulletin (Biológica! Series), v. 17, n. 4,40 p., 1972 .

KOHLS, C.M. Conceming the identity of Amblyomma maculatum, Amblyomma tigrinum, Amblyomma triste and A. ovatum of Koch, 1844. Proceedings of the Entomological Society of Washington, Washington, v. 58, n. 3, p. 143-147, 1956.

MACHADO, R.Z., FERREIRA, F.A., MACHADO, C.R., et al Ecologia em carrapatos XII - Boophilus micropius (Canestrini, 1887) em infestações naturais de veados (Ozotocerus bewarticus bewarticus, Linnaeus, 1766) e capivaras (Hydrochoerus hydrochoeris hydrochoeris, Linnaeus, 1762)dos Estados de São Paulo e Mato Grosso do Sul. ARS - Veterinária, v. 1, n. 1, p. 47-50, 1985.

MAURI, R., NAVONE, G.T. Ectoparasitos (Siphonaptera y Acari) mas comunes em Dasypodidae (Mammalia: Xenarthra) de Ia Republica Argentina. Revista de Ia Sociedad Entomológica Argentina, v. 52, n. 1-4, p. 121 $122,1993$.

NOWAK, R.M., PARADISO, J.L. Walker's Mammals of the Worid. 3.ed. Baltimore: Johns Hopkins University Press, 1986. $1500 \mathrm{p}$

SERRA-FREIRE, NM, TEIXEIRA, R.H.F., AMORIM, M., et al. Amblyomma tigrinum parasita do Cervo do Pantanal Brasil associado a Boophilus micropius. Arquivos da Sociedade de Zoológicos do Brasil, (CONGRESSO BRASILEIRO DA SOCIEDADE DE ZOOLÓGICOS DO BRASIL, 18.), Sorocaba, SP, n. 14-16, p. 50, Anais..., 1995. Ill p.

SCHULZE, P. Neves über Brasilianische Amblyommen. Zoologischer Anzeiger, v. 134, n. 5-6, p. 93-104, 1941.

SILVA, F., Mamíferos Silvestres - Rio Grande do Sul, 2. ed., Porto Alegre: Fundação Zoobotânica do Rio Grande do Sul, 1994. 246 p., (Publicações Avulsas FZB, n. 7).

SILVA NETO, P.B. Criação racional e comercial de animais silvestres. Jornal SÓS Mata Atllântica, São Paulo, SP, mar./abr.1995. Ano 7, n. 2, p. 3.

VOGELSANG, E.G, CORDERO, E.H., Lãs garrapatas (Ixodidae) de Venezuela. Revista de Medicina Veterinária y Parasitologia, v. 2, n. 1-2, p. 71-75, 1940.

VOGELSANG, E.G., SANTOS DIAS, J.A.T, Nueva contribuicion ai estúdio de Ia fauna ixodológica de Venezuela. Revista de Medicina Veterinária y Parasitologia, v. 12, n. 1-4, p. 63-89, 1953. 